\title{
Biomechanical modelling of the upper limb for robotics-based orthotic tremor suppression
}

doi:10.1533/abbi.2004.0038

\author{
E. Rocon, A. F. Ruiz and J. L. Pons \\ Instituto de Automática Industrial, CSIC, La Poveda, 28500 Arganda, Madrid, Spain
}

\begin{abstract}
Orthotic management has been proposed as an interesting alternative to current tremor management methods. It is expected that an improvement on manipulative function can be obtained by reducing the tremorous motion associated with some neurological disorders. For this to be possible, a sound modelling of the tremor and the biomechanical characteristics of the upper limb is required. This paper proposes a model for both the tremor motion and the biomechanical parameters of the upper limb. Based on these models and on a prototype of a robotics-based active orthosis two tremor reduction strategies are proposed.
\end{abstract}

Key words: Tremor, rehabilitation robotics, orthotic.

\section{INTRODUCTION}

Tremor is a rhythmic, involuntary muscular contraction characterized by oscillations (to-and-from movements) of a part of the body (Anouti and Koller 1998). Although the most common types of tremor were subject to numerous studies, their mechanisms and origins are still unknown. The most common of all involuntary movements is tremor, which can affect various body parts such as the hands, head, facial structures, tongue, trunk and legs; however, most tremors occur in the hands.

Tremor is a disorder that is not life-threatening, but it can be responsible for functional disability and social embarrassment.

More than $65 \%$ of the population with upper limb tremor present serious difficulties while performing daily living activities (Rocon et al 2004). In many cases, tremor intensities are very large, causing total disability to the affected person. There is no known cure for a lot of tremor diseases. The overall management is directed towards keeping the patient functioning independently as long as possible while minimizing disability. In addition to medication, rehabilitation programmes and deep brain stimulation, biomechanical loading has appeared as a potential tremor suppression alternative. Biomechanical loading relies on an external device that either passively or actively acts mechanically inparallel to the upper limb.

Corresponding Author:

J. L. Pons

Instituto de Automática Industrial

CSIC, La Poveda, 28500 Arganda, Madrid, Spain

Email: jlpons@iai.csic.es
Significant results have been obtained in reducing hand tremor by applying mass, friction and viscous resistive forces.

There are essentially two main approaches to reduce tremor (Koller 1987), first, isolation of the task from the tremorous limb and second, absorption of the tremorous movement. Task isolation of the tremorous limb could be done by filtering both mechanically or electronically. The issue of physically isolating the task from the tremorous limb has been attempted in the context of tremor in patients with ataxia. It involves the manipulation through a series of linkages (conceptually similar to suspensions) or using systems that execute the task (Kotovsky and Rosen 1998).

The concept of electronically isolating a task has been largely used in the microsurgery research area. The two approaches available for this application are either filtering the command signal during teleoperation or active oscillation control. Activities such as driving a wheelchair, manipulating a rehabilitation robot or accessing a computer require a standard input device (mouse, joystick). In this case, the goal is filtering out tremor-related frequencies in the tracking signal obtained from input devices when used by patients affected by pathological tremor, generating an intermediate signal, which is sent to the controlled subsystem (wheelchair, robot arm or cursor).

The approach of absorption of the tremorous movement will be briefly introduced in the next section. This is the approach used in dynamically responsive intervention for tremor suppression (DRIFTS) project (Manto et al 2003).

\section{Absorption of the tremorous movement and tables}

The effects of load and force on tremor have received considerable attention by the research community. Among 
others, Adelstein (1981) has conducted a thorough analysis of the effect of viscous loading as a means for active reduction of intention tremor. As a result, Adelstein reports that significant and steady reductions of tremor amplitude are observed as the viscous loading increase. This phenomenon gives rise to the possibility of an orthotic management of tremor.

Tremor absorption is often confused with tremor isolation. The approach of tremor absorption, however, is based on tremor reduction devices that act mechanically parallel to the oscillating limb. They are energy dissipaters that apply a shunt load between the limb and a fixed reference frame.

One of the specific and important common aspects to the field of orthotic rehabilitation is the intrinsic interaction between human and robot. This issue, in its simplest manifestation, implies a mechanical interaction between the robot and the human, most often solved through impedance control approaches (Harwin et al 1998). The basic principles and considerations regarding impedance control were addressed by Hogan (1985) in an excellent work. In his paper, Hogan pointed out the conditions for causality in the treatment of dynamic interaction between manipulator and environment, used the concept of mechanical impedance to address the mechanics of muscular skeletal system, dealt with the implementation of this control approach, and eventually, addressed the selection of an appropriate impedance for a given application.

Biomechanical loading for tremor reduction can be approached either by ambulatory robotics-based orthotic devices or by non-ambulatory table or wheelchair mounted devices. The former approach is characterized by selective tremor suppression through internal forces at particular joints, while the latter relies on global application of external forces that leads to the overall tremor reduction. In Figure 1(a), a table-mounted tremor suppression device is shown (Neater Eater), which is an example of mechanisms implementing the external force concept for reducing tremor. Figure 1(b) shows the internal force concept, a wearable exoskeleton device applies tremor cancelling forces between upper-limb segments (the WOTAS-DRIFTS device).

Although wearable tremor suppression exoskeletons are already a matter of research, non-ambulatory systems have lead to commercial products; see for instance, the socalled Neater Eater (Michaelis 1988). In addition, the MIT damped joystick (Hendriks 1991), the controlled energydissipation orthosis (CEDO) (Rosen et al 1995), or the modulated energy dissipation arm (MED) (see (Kotovsky and Rosen 1998)), are implementations of non-ambulatory, wheelchair mounted tremor-suppression prototypes. As far as wearable tremor suppression concepts are concerned, just the well-known wearable tremor-suppression orthosis (Kotovsky and Rosen 1998), has been reported in the literature. This is a passive damping-loading device, which acts mechanically inparallel to the wrist in flexo-extension.

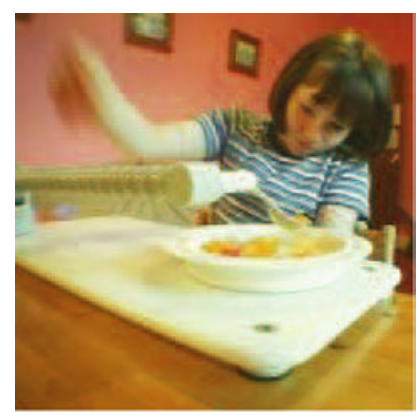

(a)

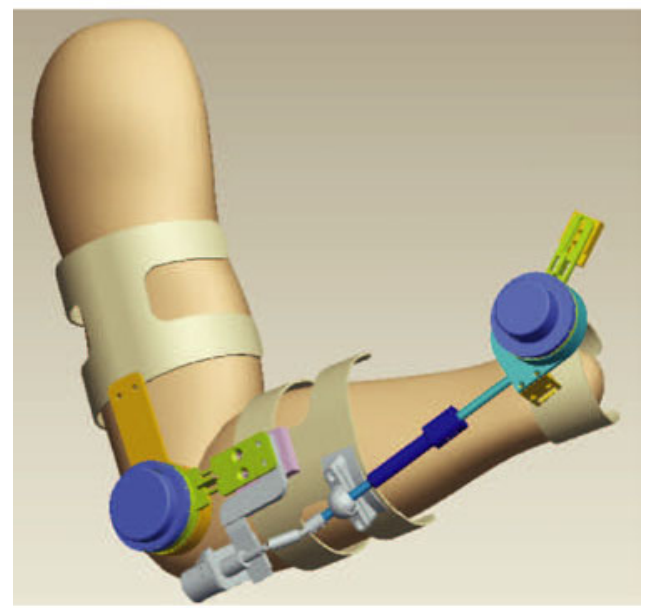

(b)

Figure 1 Typical examples of (a) non-ambulatory tremor suppression mechanism (Neater Eater) and (b) orthotic device for tremor reduction (WOTAS).

It completely constrains both wrist abduction-adduction and prono-supination.

For a successful active tremor absorption mechanism, a means for intelligent detection of tremor versus voluntary motion is required. To this end, a model of the tremor motion must be proposed. In the following sections, a sinusoidal model for the tremor is used. Based on this model, a two-stage modelling approach is used to detect tremor and voluntary motion, see section "Modelling of Tremor". In section "Modelling the Tremorous Limb", the upper limb is modelled as a second-order biomechanical system. This, in combination with the tremor model from section "Modelling of Tremor" allows the implementation of control strategies.

\section{MODELLING OF TREMOR}

It is clear that both approaches will depend strongly on the quality of our estimation of tremorous and voluntary motion. A number of estimation algorithms have been developed for tremor suppression. As a first approach, we used robust algorithms based on the IEEE-STD-1057 Standard. In particular, the weighted-frequency Fourier linear combiner (WFLC) developed by Riviere in the context of actively counteracting physiological tremor in 


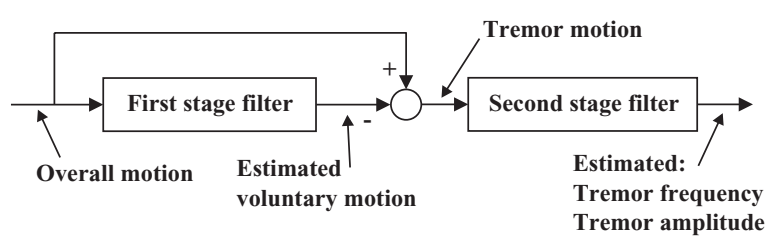

Figure 2 Two-stages of tremor modelling: first, the low frequency content voluntary motion is estimated, second, the voluntary motion estimation is subtracted from the original motion, eventually, tremor frequency and amplitude are determined.

microsurgery. The WFLC is an adaptive algorithm that estimates tremor using a sinusoidal model, estimating its time-varying frequency, amplitude and phase (Riviere 1995). The main problem of using the WFLC is that it requires a previous filter stage in order to remove the voluntary motion of the overall movement.

This filter stage introduces a time delay that could considerably affect the implementation of the control strategies proposed in the previous section for tremor suppression.

The ideal solution is the development of an algorithm capable of estimating voluntary and tremorous motion without phase lag. It is well known that the frequency of the voluntary motion of activities of daily living, ADL, occurs at frequencies lower than the tremorous movements (Riviere 1995). Based on this statement, we have developed a new algorithm comprising two stages that could offer a satisfactory solution for this problem (Figure 2).

In the first stage, the voluntary motion is estimated based on an implementation of the above-introduced WFLC algorithm. This stage does not consider any movement of high frequency, particularly spasms, which are very common in patients who suffer from neurological disorders. Spastic movements are considered as tremorous movements and thus, the control strategies would try to filter out and reduce their amplitude.

In the second stage, the estimated voluntary motion is removed from the overall motion and the assumption that the remaining movement is tremor is made. After this, we use the IEEE-STD-1057 Standard in order to estimate the tremor. In this stage, the algorithm estimates both the amplitude and the time-varying frequency of the tremorous movement.

To evaluate the performance of the algorithm, some voluntary movements were simulated in wearable orthosis for tremor assessment and suppression (WOTAS), the exoskeleton platform of Figure 1(b). WOTAS is an active orthotic device. It includes active elbow flexionextension, active forearm pronation-supination and active wrist flexion-extension, see Figure 1(b) for a schematic view. We have evaluated this algorithm with data obtained from 33 patients suffering from different tremor diseases. The estimation error of first stage was $1.0359 \pm 1.3816$ degrees. The second stage algorithm has a convergence time always smaller than $2 \mathrm{~s}$ for all signals evaluated and the

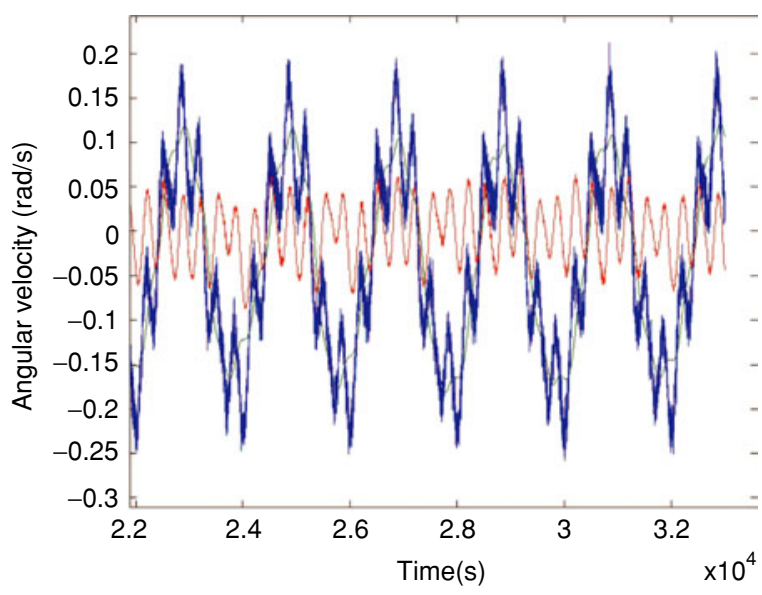

Figure 3 Modelling of tremor as a sinusoid: velocity signals (blue), estimation of voluntary movement (black dotted) and estimation of tremor (red).

MSE between the estimated tremor and the real tremor, after the convergence, is smaller than the 1 degree. The combination of both techniques resulted in a very efficient algorithm with less processing cost for estimating real time of the voluntary and the tremorous components of the overall motion.

Figure 3 illustrates the performance of the algorithm when estimating the voluntary movement (simulated by a low frequency sinusoidal movement) and the tremorous movement (simulated by a noisy high frequency sinusoidal movement).

\section{MODELLING THE TREMOROUS LIMB}

\section{Control strategies}

As already pointed out, both ambulatory and nonambulatory concepts can be implemented though passive and active systems. In active systems (Michaelis 1988, Rosen et al 1995), active actuators generate an equal but opposite motion based on a real-time estimation of the involuntary component of motion, actively compensating and effectively subtracting the tremor for the overall motion. In passive concepts, a mechanical damper is used (Kotovsky and Rosen 1998), thus the dissipative force usually results from shear forces at the damper's fluid. One of the main drawbacks of passive system is that the dissipative force is also loading the patient's voluntary motion. As a consequence, the user feels a mechanical resistance to the motion. Even though in active systems this could be avoided, to the author's knowledge, the prototypes reported in the literature do not filter out voluntary motion, and thus, the dissipative force also affects voluntary motion.

In this section, we propose two different control strategies based on biomechanical loading to suppress tremor:

(1) Tremor reduction through impedance control: implements an impedance control, i.e. the stiffness, damping and 


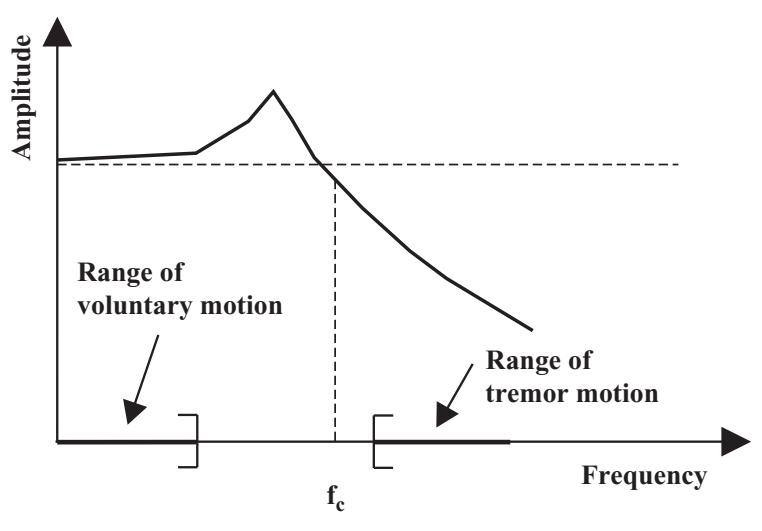

Figure 4 The musculo-skeletal system is modelled as a second order biomechanical system. The active orthosis is used to modify the apparent biomechanical characteristics of the upper limb so that the cut-off frequency, $f_{\mathrm{c}}$, lies between the frequency range of voluntary and tremor motion.

mass properties (corresponding to a second order model of the musculo-skeletal system) of the upper limb can be modified to study its effects on tremor.

(2) Notch filtering at tremor frequency: based on noise reduction techniques, implements an active noise filter at the tremor frequency taking advantage of the repetitive characteristics of tremor.

\section{Modification of the second-order model}

The impedance of a system can be defined as a relationship between the reaction force of the system to an imposed external motion and the motion itself. In general, impedance comprises three components, i.e. stiffness, damping and mass. There is evidence (Adelstein 1981) that all the three components modify the biomechanical characteristics of tremor at the upper limb, which in general can be described by a second-order system.

The DRIFTS control scheme is conceived so that the effect of the suppression load on voluntary motion is minimized. The big challenge in this approach is to distinguish error from intended motion before error cancelling can occur. This requires real-time error estimation.

In this approach, the musculo-skeletal system (each upper limb articulation contributing to tremor) is modelled as a second-order biomechanical system. It is well known that the frequency response of a second-order system exhibits a low-pass filtering behaviour. The cut-off frequency of this second-order system is directly related to the biomechanical parameters of the second-order system, i.e. inertia, damping and stiffness. Our approach consist in selecting the appropriate modified values of inertia and damping of the musculo-skeletal system so that the cut-off frequency lies immediately above the maximum frequency of the voluntary motion and well below the tremor frequency, see Figure 4.

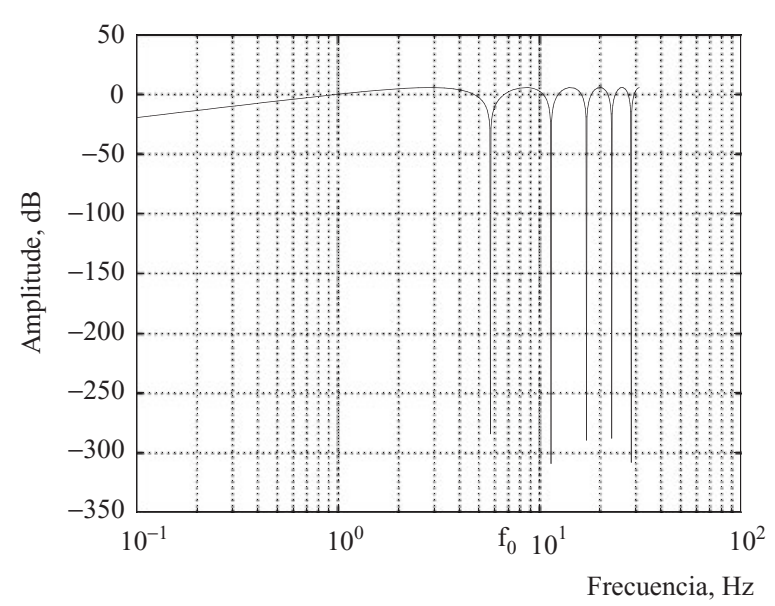

Figure 5 An active notch filter is implemented by means of the active orthosis. In this scheme, the notch filter frequency, $\omega_{0}$, lies exactly at the tremor frequency.

\section{Exploiting the repetitive characteristics of tremor}

Tremor is usually defined as rhythmic, involuntary muscular contraction characterized by oscillations at central frequency (Anouti and Koller 1998). Tremor frequency varies according to the particular neurological disorder being considered. In particular, while essential tremor takes place in the frequency range between 5 and $8 \mathrm{~Hz}$, rest tremor is usually found at a slightly lower frequency range $3-6 \mathrm{~Hz}$.

In addition, for a given type of tremor, its main frequency varies from patient to patient, but tends to be quite stable for a particular subject. This property should be exploited when designing a control strategy to counteract tremor. In particular, repetitive control can handle periodic (repetitive) signals and disturbances. Repetitive control can be regarded as a subset of learning control since the control action is determined using the stored error values from preceding periods. Even though repetitive approaches can handle periodic signals (tremor), it is not free from some common problems: tight stability conditions, poor response to non-periodic and non-harmonic signals and poor noise characteristics (Inoue 1990).

The idea behind this control strategy is that active actuators generate an equal but opposite motion, based on a real-time estimation of the involuntary component of motion, actively compensating and effectively subtracting the tremor for the overall motion, see Figure 5. As in the previous control approach, the quality of the tremor suppression control approach is strongly dependent on accurate estimation and tracking of tremor frequency.

\section{CONCLUSIONS}

This paper presented a modelling approach for both the characteristics of tremorous motion and the upper limb musculo-skeletal system. Both models have been used to implement an orthotic active tremor suppression approach. 
The proposed method aims at modifying the intrinsic biomechanical, inertial and damping characteristics of the upper limb so that tremor is reduced. This approach is found to be very sensitive to the accurate estimation of tremor characteristics, i.e. amplitude and frequency. In order to solve this problem, a tremor estimation algorithm that can work in real time has been developed.

The system has been subjected to experimental verification on an active exoskeleton orthotic device. The tremor estimation has resulted in accurate prediction of tremor motion without significant delay. The system is thus ready for starting first pre-clinical trials.

\section{ACKNOWLEDGMENT}

The work presented in this paper has been carried out with the financial support from the Commission of the European Union, within Framework 5, specific RTD programme "Quality of Life and Management of Living Resources", Key Action 6.4 "Ageing and Disabilities", under contract no. QKL6-CT-2002-00536, "DRIFTS - Dynamically Responsive Intervention for Tremor Suppression".

\section{REFERENCES}

Anouti A, Koller W. 1998. Tremor disorders: diagnosis and management. West 7 Med, 162(6):523-30.

Rocon E, Belda-Lois JM, Sanchez-Lacuesta JJ, Pons JL. 2004. Pathological tremor management: Modelling, compensatory technology and evaluation. Technol Disability, $16: 3-18$.

Koller WC. 1987. Handbook of Parkinson's Disease, New York: Marcel Dekker.

Kotovsky J, Rosen MJ. 1998. A wearable tremor suppression orthosis. 7 Rehabil Res Dev, 35(4):373-87.

Manto M, Topping M, Soede M et al. 2003. Dynamically responsive intervention for tremor suppression. IEEE Eng Med Biol, 22(3):120-32.

Adelstein BD. 1981. Peripheral mechanical loading and the mechanism of abnormal intention tremor, PhD thesis, MIT.

Harwin WS, Leiber LO, Austwick GPG et al. 1998. Clinical potential and design of programmable mechanical impedances for orthotic applications. Robotica, 16:523-30.

Hogan N. 1985. Impedance control: An approach to manipulation: Part I, II and III. F Dyn Syst, Meas Control, 107(1):1-23.

Michaelis J. 1988. Introducing the neater eater. Action Res, $6(1): 2-3$.

Hendriks J. 1991. A second-generation joystick for people disable by tremor. Proceedings of the 14th Annual RESNA Conference, Elmsford, NY, Arlington, VA: Resna Press, pp. 248-51.

Rosen MJ, Arnold AS, Baiges IJ et al. 1995. Design of a controlled-energy dissipation orthosis (CEDO) for functional suppression of intention tremors. 7 Rehabil Res Dev, 32(1):1-16.

Riviere C. 1995. Adaptive suppression of tremor for improved human-machine control, Ph.D. thesis, Johns Hopkins University.

Inoue T. 1990. Practical repetitive control system design. Proceedings of the 29th IEEE Conference on Decision and Control, Vol. 3, pp. 1673-8. 

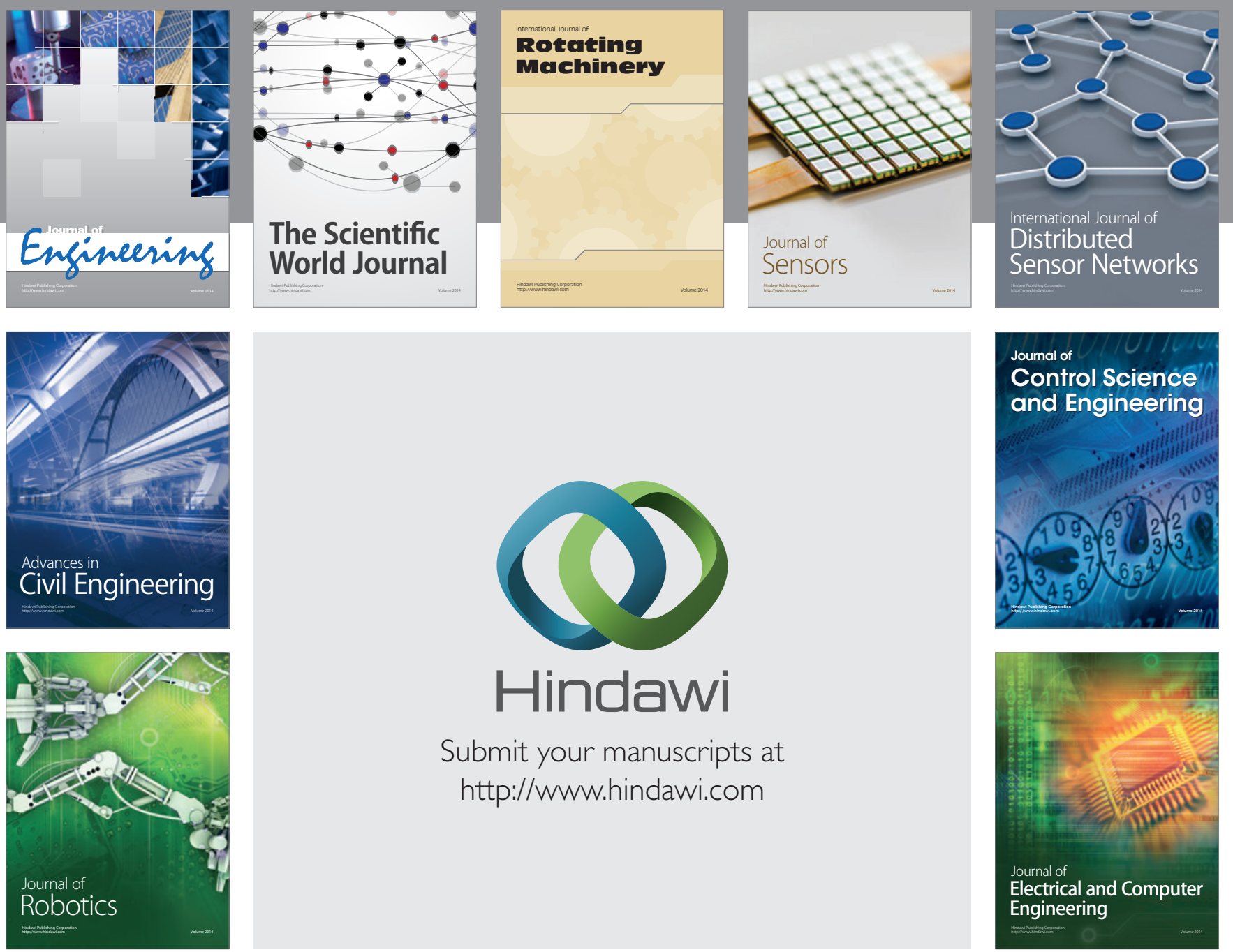

Submit your manuscripts at

http://www.hindawi.com
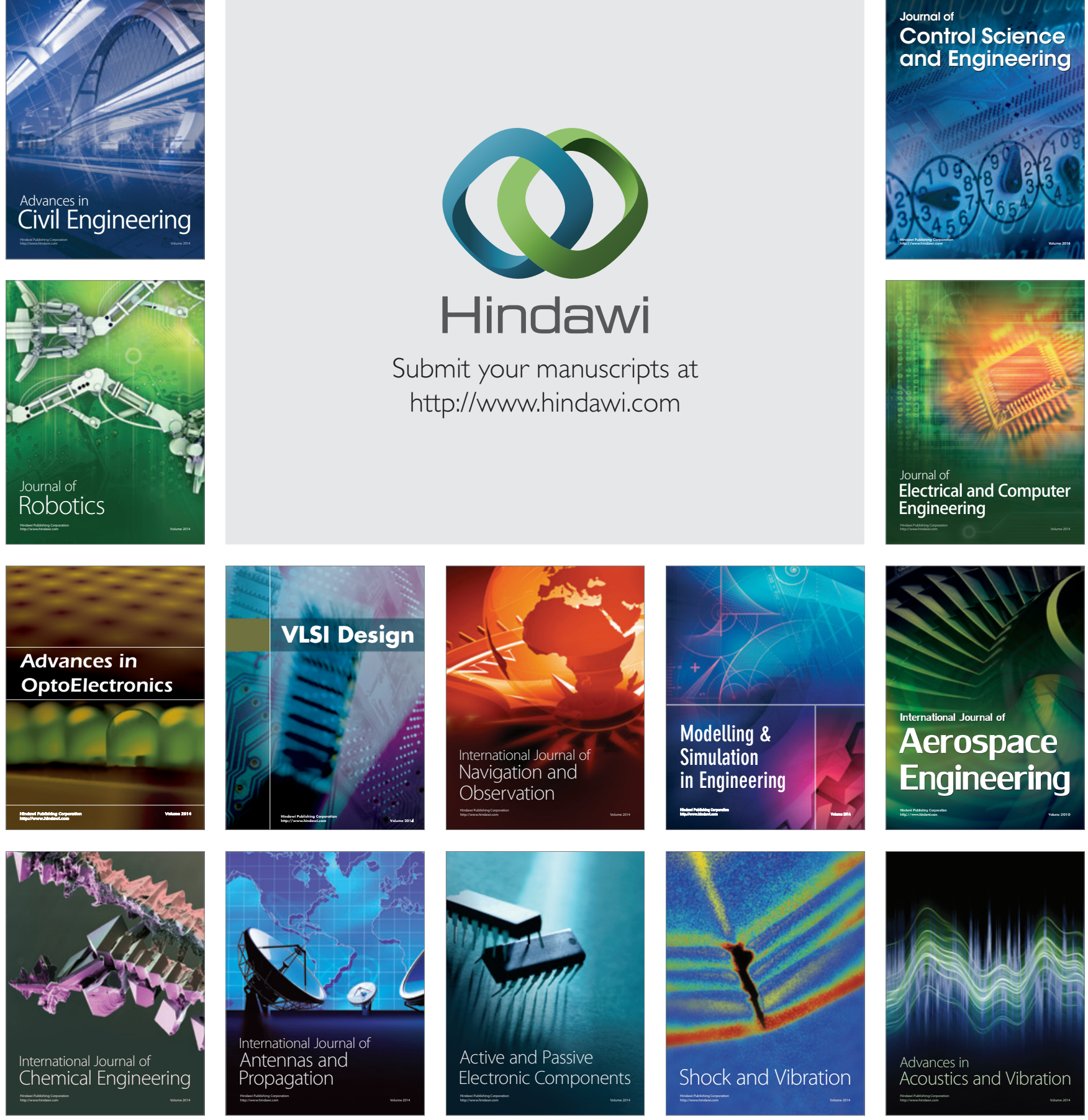